\title{
Body Language: Symbiosis Between Art, Culture and Animated World
}

\author{
Dahlan Abd Ghani, Syed Hassan Bin Syed Omar
}

\begin{abstract}
Inside the world from movies, it's very important to apply the body language of each part of characters. The purpose of this research is to study the important and ways of robotic body language in animated film. This research also will focus on the important of mood and body language related to movement in Malaysia's animated series which is can make a way to look in deep of visual storytelling, art of acting and others technical sequences that involve when making an Animated Short Film. Thus, from this research a prototype short animated film known as "SpiDay" will be produce by using $3 D$ animation media technique.
\end{abstract} language, mood, acting, $3 D$.

\section{INTRODUCTION}

Today's into the future, producing and creating animation make an opportunity for animators by doing different type of techniques, style, theme, experiment and stroke for produce an animation. During the period of time there are a lot of changes through major or minor has risen as technology growing rapidly. From ice age of animation until three dimensional animations there are a lot of changes in this part. Animation is the process when the panel keep rapidly change to make a sequence of static image that move in a period of time. For now, animation can be manipulated easier by using software. There's a lot of techniques for recorded an animation such as a flip book, flash animation, video recorded tape, digital media, animated GIF formats, and many more. It's doesn't matter what technique that we use, the main objective is the principal that we can apply in our animation. A lot of ways to serving animation, from small inch of screen to the largest screen we can project into it, the technological development helps the world of animation growth rapidly too.

Body language is a form of mood in each character that we have developed into different characters. In the simple angle of term, body language is the forms that make characters look come to life. The body language is the main part to show the characteristic of characters, such as the movement of characters, expression of feeling, how they talk, happy, angry and so on. If we don't apply body language into the characters, we can be lack of mood from the story and can't feel the characters. Body language is one of the important elements that show everything in film and animation. This is some example of brilliant and good body

Revised Manuscript Received on September 10, 2019.

Dahlan Abd Ghani, University Kuala Lumpur, Malaysian Institute of Information Technology, Jalan Sultan Ismail, Kuala Lumpur, Malaysia.

(Email: syedhassanalattas90@gmail.com)

Syed Hassan Bin Syed Omar, University Kuala Lumpur, Malaysian Institute of Information Technology, Jalan Sultan Ismail, Kuala Lumpur, Malaysia

(Email: dahlan@unikl.edu.my)
Keywords: robotic, animated short films, animation, body

language can be feel in such film as; Alice in wonderland (1951), Toys story II (1999), Transformers series (19842015), beuty and the beast (1991), Despicable me (2010), Futurama (1999-2013), Robots (2008).

Body language is a type of non-verbal communication in which physical behaviour, as opposed to words, are used to express or convey information. Such behaviour includes, facial expressions, body posture, gesture, eye movement, touch and the use of space. This language exists in all of characteristic. Body language is a process of communication, and we can call it the second-language in living thing. The first known used is from book title "The hidden dimension (1966) by Edward T. Body language is not only use in theatre or movies, it can also be applied into animation films. The use of body language is to show the characteristic of the character and make the characters goes for real. Body language is significant to communication and relationships, it can be observed by many people thus can reveal much about feeling.

\subsection{Story Line}

This short animation named "Spiday" is a story about a robot spider walking around in dystopian city. This spider is looking for a new friend because he's felt alone and boring. Then spider hear the sound of dog. Spider go to that sound and found that's only a voice from dog in version of robot After seeing that spider feel sad and go for walking again. Spider rest while watching dystopia scenery and feel the sound of wind and all surrounding. Spider start walking back, when spider stick on wall there's an article write about corruption. After that spider meets some ball bouncing, and start follow that ball until come to the one door the ball bounces up inside backdoor. The spider tries to climb up the door to follow the ball. When spider come to top of the wall spider can't see whats behind the door, so spider move forward. End of this story is spider falling down and dies because of fall from walls. There's a lot of moral value inside this short animation.

\subsection{Medium}

For this short animation, I will be combining 3D and composition effects to have the look and scenery of the toon world. I will use colour correction for each emotion's play by scene. The medium which I will be using is mostly software based mainly Autodesk 3DsMax (for the 3d modelling and animating) and Adobe After Effects CS6 (for the compositing and effects). I will also be using Adobe Premiere Pro, Adobe Photoshop, Adobe Illustrator, and the 
3D will be rendered in Mental Ray.

\subsection{Duration}

For the short animation project, the requirements are to produce a short animation not less than three-minute length.

\subsection{Background Of Study}

This study is carried out for help to understand the ways and important of applying body language into the characters inside the shot while applying effects, setting up composition and to understand more and deep in creating and producing a short animation film and the attraction in mood that effects the quality of the final short animation itself. I am going to have a good result from my research and to understand a lot of important about body language in making a good animated film.

\subsection{Scope \& Limitation}

The purpose of working the animation is to test an experiment on the effectiveness of implying the body language and the combining animation effects which is 3D and Visual Effects. I have collected some of information about how they do apply body language plus mood in character by referring the present and past animation/film that practise the same method and type of animation. The limited resources of body language in animation at Malaysia which make it difficult to apply the level of quality in body language since they not to be fragile in the important of body language like others country.

My aim for targeting audiences and viewers is people that do work in making film, animation film, advertising and any of scope that do applying the body language before they started created their own new file in animation film.

\subsection{Significant Of The Study}

The urge of this study is wanting to study deeper and understand the major of body language really is. This will bring some first places how important and special in body language and mood can be. It's also gives and impact to animators that beauty of body language can bring the audience to have a better feeling of the story without miss any part in it.

\subsection{Conclusion}

A lot of many things to be considered when making or producing a short animation film. It does not have a specific step or stages, but it does have its own process and workflow for made work being guided in creating nor produce the short animation film with good quality standard. The use of applying body language in any animation film will make those animations can touch the hearts deeper.

Limitation is always coming in producing or creating a short animation film. It will often be about time management on how to finish the task on time because of sharp time, so there will be sacrifices made in the quality of the short animation film while try to priority the part that makes the animation special and one in the seconds.

If this research is awesomeness success, it can really show how to absorb the body language for affects the mood and quality of a short animation film.

\section{LITERATURE AND ANIMATION REVIEW}

This chapter representing a review of the literature on the important of body language in short animated film. The objective of this study was to develop a better understanding of usage in body language and Emotion effects in Animated Films. In this section, I will review some animations that influence my FYP short animation. It is with Macross Plus Movie Edition (1984), directed by Shoji Kawamori. One of the best thging about animation is that is full of robot fighting. I think, For example in a scene at the end of the film

\subsection{Introduction}

People are constantly throwing off a storm of signals. These signals may be silent (non-verbal) messages communicated through the sender's body movements, facial expressions, voice tone and loudness; we like to call it body language or sixth sense of living thing. This research focuses on robot body language. Robot locomotion is the collective name for the various methods that robots use to transport themselves from place to place. Although wheeled robots are typically quite energy efficient and simple to control, other forms of locomotion may be more appropriate for a number of reasons.

\section{Types of Robots by Locomotion}

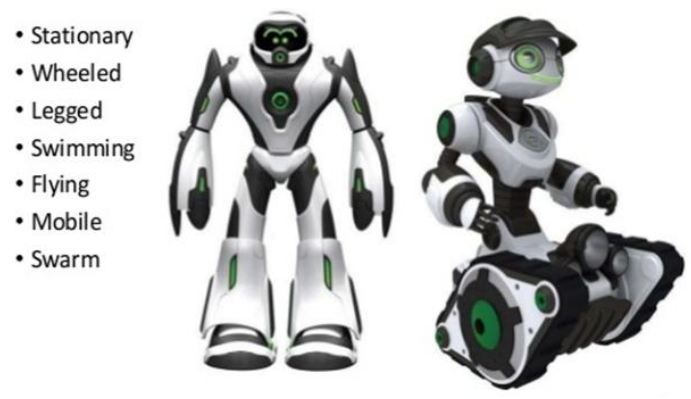

2.1 Diagram shown type locomotion of robots. Source from http://goo.gl/c7E.bt

\subsection{BODY LANGUAGE IN ANIMATED FILMS}

Body language in animation places an important role in showing the mood of the characters. Using difference body language in difference situation can result a good emotional or role very nice. Mood of audience is to show if the body accomplish or not, this is challenging stages for animation to attract with audience emotion plus the good role of the story is "show but don't tell".

A good example of a character with expressive body language is Pingu. He has very over the top body movements to show his hyperactive personality. Often, he moves in ways which Penguins can't, such as stretching his beak. This just expresses his feelings and reinforces his cartoonish character. ( Pingu, 1986) 


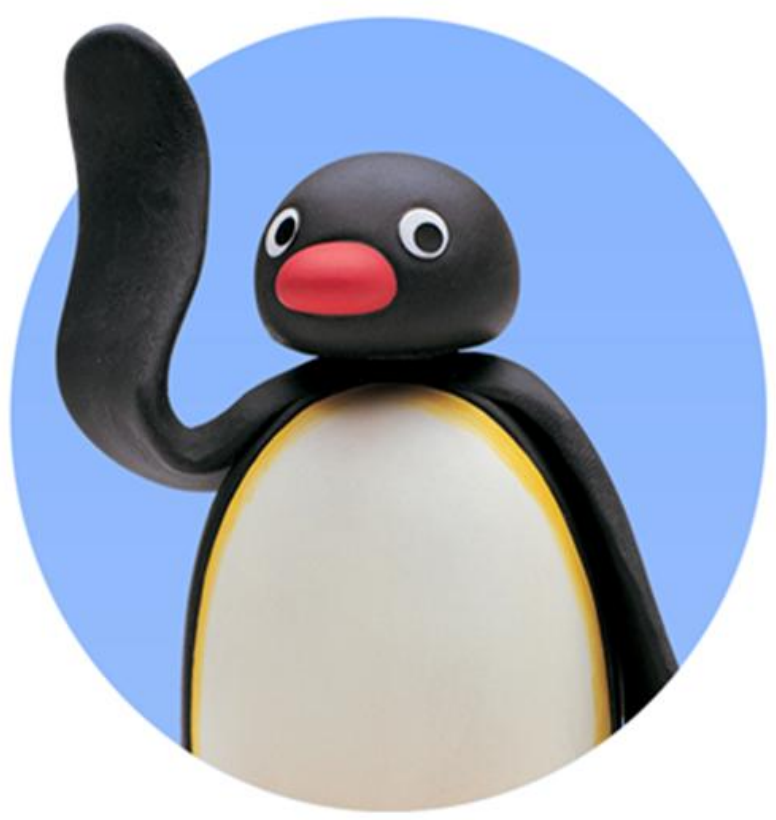

Figure 2.2: Source: “Pingu”by Tommonmon pages.

\subsubsection{DIFFERENT TYPE OF BODY LANGUAGE}

A body pose in each part of the scene is different as the mood of the character. Some character plays sad feeling some other's beside of that. As a result, in silhouette we currently can feel or see the different of body language that each characters bring for making different and makes characteristic or mood receive clearly. Here are some example silhouettes of body language/body gesture.

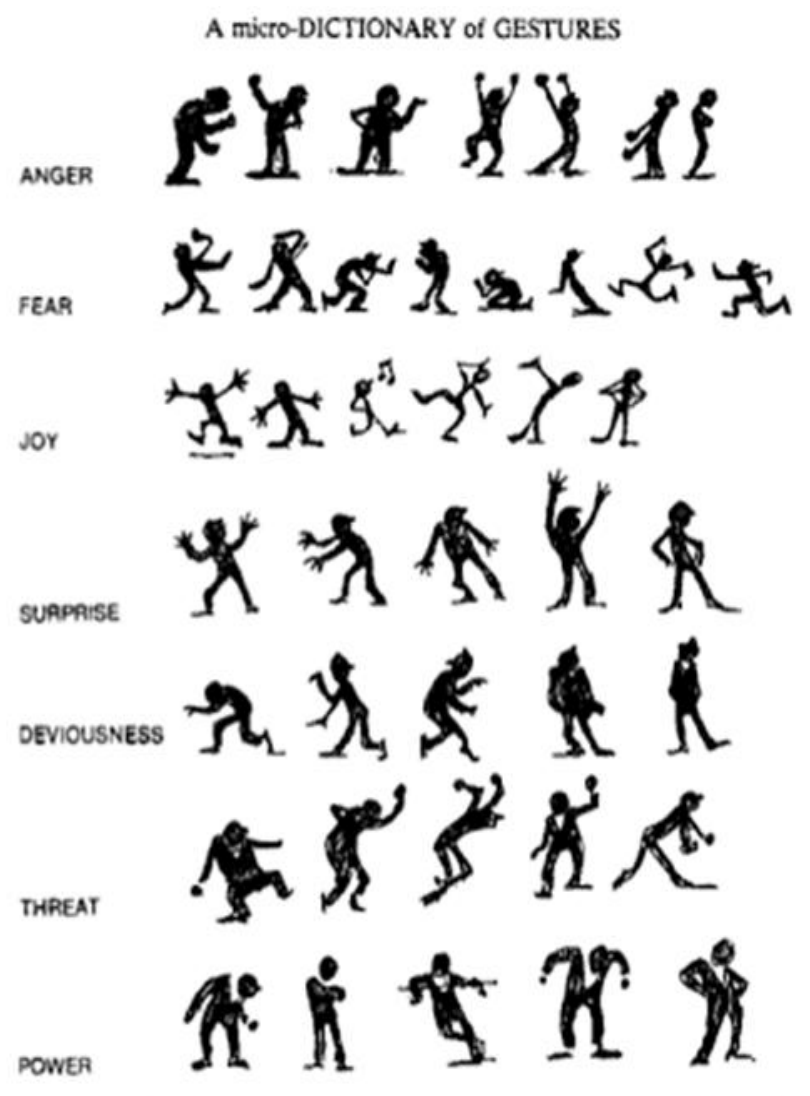

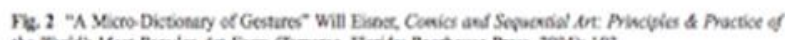

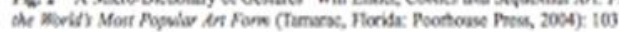

Figure 2.3: Source: "Body language in silhouettes" by Tomnomnom.
Every part of characteristic in each character have their gesture guide line for making a perfect body language. Each body language is full of emotion as we know when we are happy then we are jump and when we are sad, we just sit lonely with facial expression.

\subsubsection{CHANGE OF EXPRESSION}

Disney master animator "Eric Larson" said he's discovered body language is one of "illusion of life". He found this while making Mickey mouse and mark this principle when using change of expression, the process of body language is showed. Change of expression is between images or emotion before its show the true emotion.

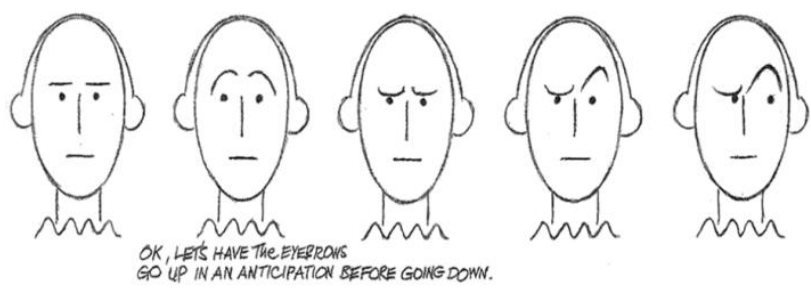

Figure 2.4: Source: "Expression changing" by Eric Larson

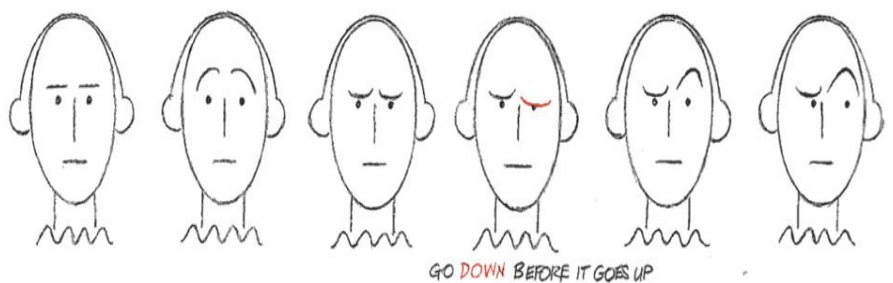

Figure 2.5: Source: "Expression change" by Eric Larson.

"The more extra position or movement make the change more and more bang for the buck"

\section{- " Eric Larson"}

\subsubsection{LOOK FOR CONTRAST}

There is movement to show the flow. During a broad move don't change the expression. It's because when we are doing something like that, the movement of animation not too smooth and make misplaces of the emotion and body language of characters in animation.
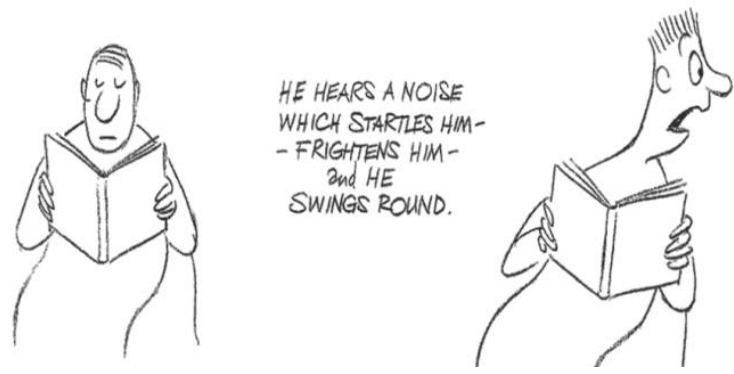

Figure 2.6: Source: "Expression during aboard move" by Milt Kahl. 
For a good start we do need something to change fromsomething opposite- Something that a lot different from that we are going to change to. The point is we don't want to change character expression during the move where we cannot see it. It's because we insert a position where we see the Change before the move. Except the body movement we need to apply it to make realistic while character ready to change into true emotion.

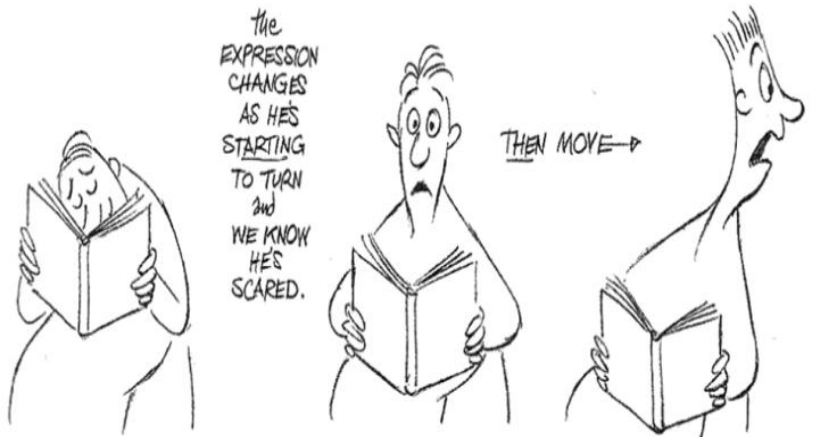

Figure 2.7: Source: "Expression during aboard move" by Milt Kahl.

\subsubsection{An Acting Point}

Still same as before here we must put the secondary action or hang timing before show some movement or some body language. The main cause is because animator must to show the smooth process of characters to had some emotion inside it. The centre nor between frame or movement is the good places for making this silent language move perfectly.

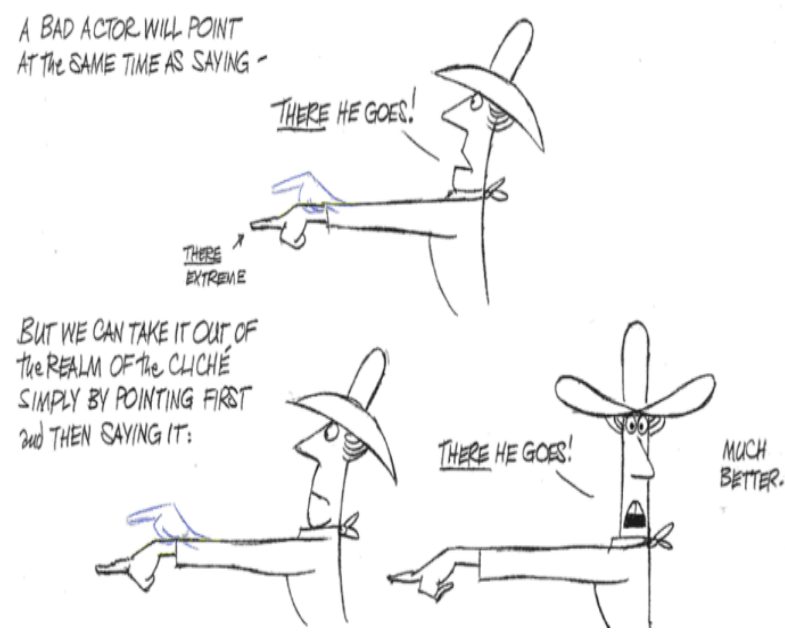

Figure 2.8: Source: “Acting point” by Milt Kahl.

This is example of finger pointing in acting point

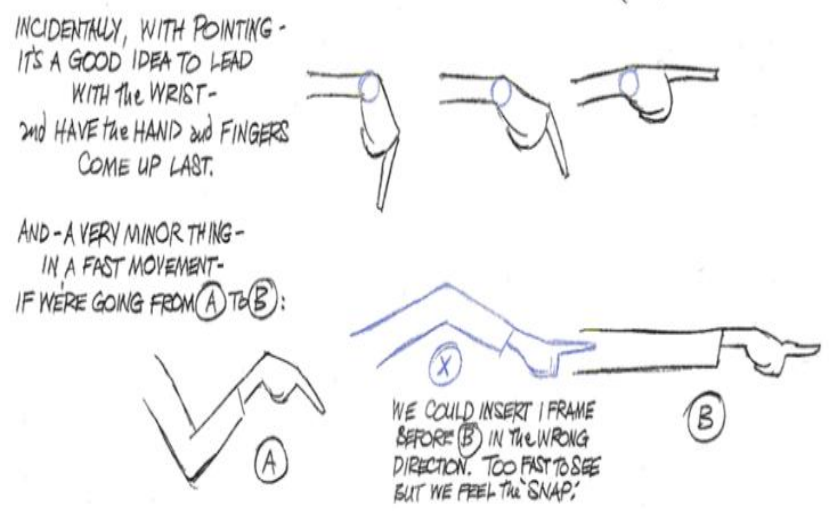

Figure 2.9: Source: “Acting point” by Milt Kahl.

\subsubsection{Body Language}

Ken Anderson said, "pantomime "is the basic art of animation. Body language is the root and fortune ely it is universal. "The Audience won't understand if we just put only the word that is why body language is so important. With body language and emotion, we should keep words to a bare minimum and make everything as clear as we can through pantomime. We must mindset that only the body will tell a good story. its means before we put those word inside animation, the body language is the first thing that we must look deeply. The clear of movement makes story flow pretty.
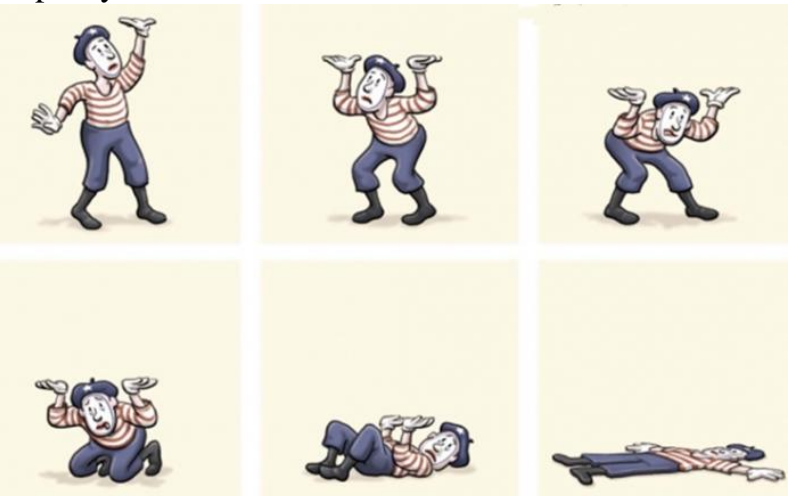

Figure 2.10: Source: "Body language" by Ken Anderson.

\subsubsection{Symmetry Or Twinning}

The Symmetry has got a bad press because of bad animation acting. People say avoid where both arms and hand are doing the same thing, that is what we called 'twinning' two movement in one situation. As an example is the politician, when they are laying down the law their arms will twin symmetrically.

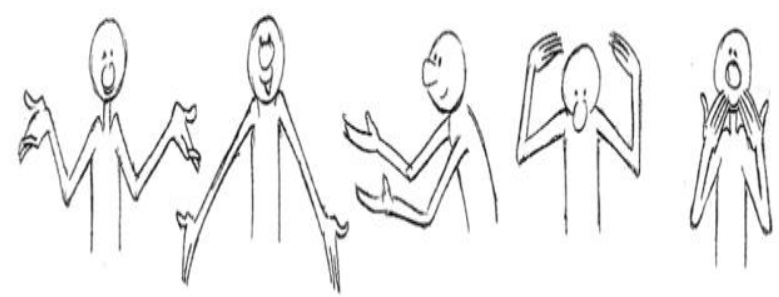

Figure 2.1.1: Source: “Body language” by Ken Anderson.

Sometime their do in reduced version, we can see how pretty this acting to be after complete using body language plus expression.

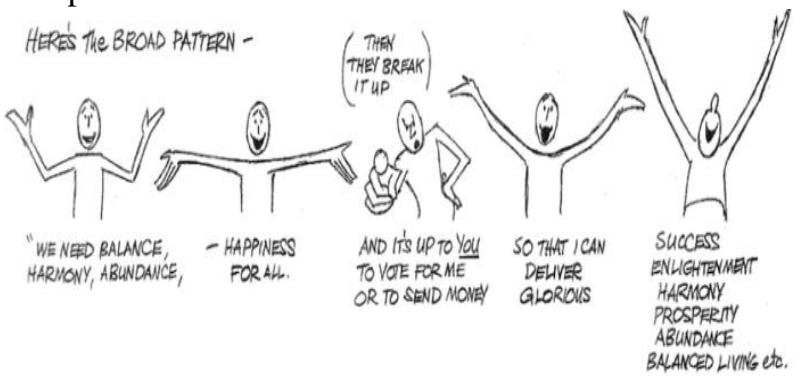

Figure 2.1.2: Source: “Body language” by Ken Anderson.

Published By: 
Symmetry is an expression of harmony, beauty, balance and people use it all the times. So, a judicious use the twinning is effective because its everywhere.

\subsubsection{Eyes}

Eye's movement can grab people attention. with eye's can show many emotion and facial expression. This part is light version to use for show the silent language. The eyes are the visible part of brain directly connected to it. The soul of person revealed in the eyes.

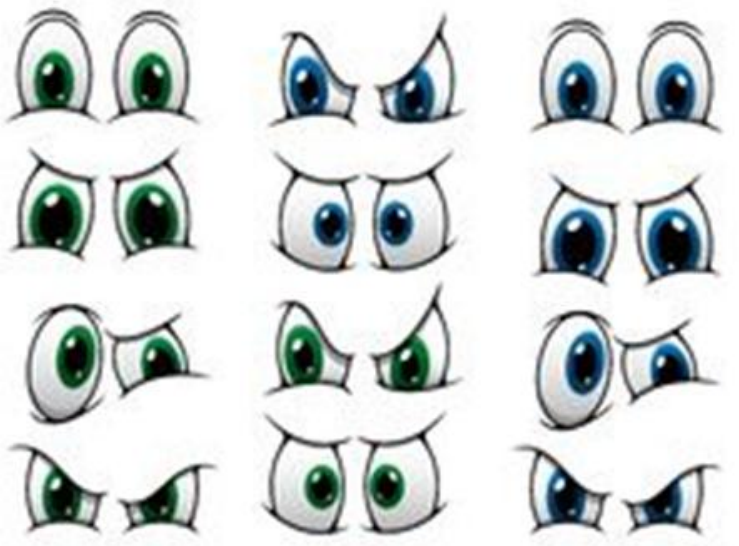

Figure 2.1.3: Source: "Body language" by Ken Anderson.

\section{RESEARCH METHODOLOGY \& RESULTS}

\subsection{INTRODUCTION}

This topic will cover the details explanation that is being used to make this project complete and working well. The method is use to achieve the objective of the project that will accomplish a awesome result. In order to evaluate this project, the methodology is based on Pre-production, Production and Post-production that consist of each stages which are requirements, design, and development. In this chapter I will show the method that will use. This report will be recording the process of doing this short animation.

"My final two cents worth of advice is to develop an allconsuming curiosity for things both exotic and ordinary. Read, observe, analyze, and become involved with a variety of interests. Study, practice, delve, probe, investigate, and above all, be flexible. Keep an open mind. The world is changing fast. Don't get caught in the corner of the ring. Ward Kimball"

\subsection{RESEARCH METHODOLOGY STRUCTURE}

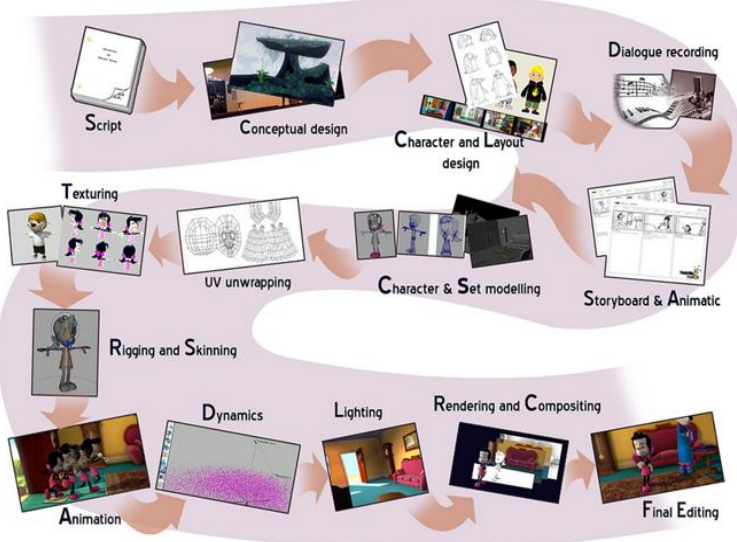

Figure 2.1.3: Methodology Structure

\subsubsection{Pre-Production}

This is planning stage. It's a part where the idea/concept, story, character design, thumbnails and storyboard to be move forward. Pre-production is the process of fixing some of the elements involved in a film, play, or other performance. There are three parts in a production: preproduction, production, and post-production. Pre-production ends when the planning ends and the content starts being produced.

Having a strong concept is an major component of the story. But unfortunately, spending the appropriate amount of time in pre-production to nail down the animation's concept is often an overlooked step in the production process.

\subsubsection{Production}

For 3D animated films, this is where the process of the modelling of character, texturing, props and rigging is done.

During the production stage, the process of animation begins. This is usually the longest stage, hardest and also the busiest of them all. This is where supporting objects (props), environments, motion, texturing, colour and lighting are suitably created.

\subsubsection{Post-Production}

3D modelling and animation services in the post production phase involve exporting and rendering of animation frames. Pieces of animation such as body language, mood are edited together and soundtrack including sound effects and voice-over incorporated. Creation of transitions, compiling, exporting and video file compression is done during this stage. Compositing, visual effects and colour correction are also handled in the post production stage.

\subsubsection{Data Collection}

This stage is a stage where area of study. It's about the projects, time, resources, references and literature studies to get more information of study. All the materials are collected from journal, texts book, article and information that on internet such as Wikipedia and so on that related in my field.

This research has done all of my project related, which includes hanging time with animation clips and videos in the NET for taking information in body language from old to nowadays animation. The good research started from Alice in wonderland the old version cartoon (1951) that they study properly in body language.

\subsubsection{Hardware \& Software Requirement}

This is the requirement of hardware and software that I am going to use during the production of the project: 
HARDWARE

\section{SOFTWARE}

PLUGIN

NVIDIA METAL RAY

REDGIANT MAGIC LOCK

Table 3.1: Hardware, Software and plugins used for the short-animated film "RobDay"

\subsection{Research Figure}

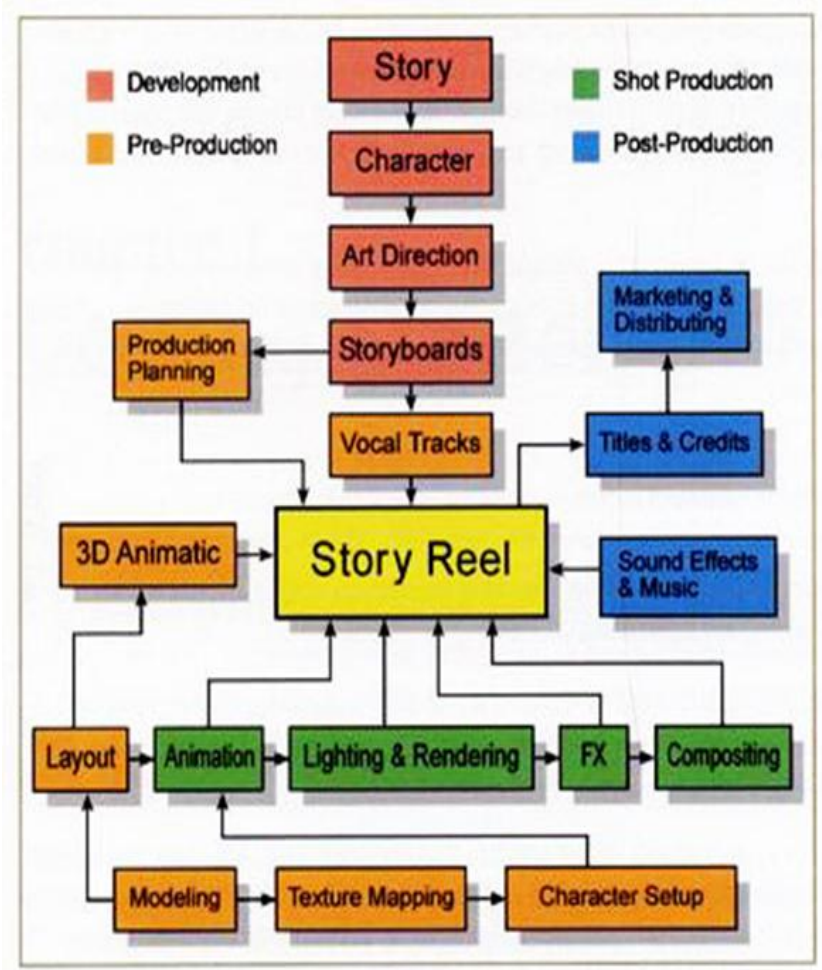

Table 3.2: "RobDay" Animation Pipeline of PreProduction, Production and Post-Production Stages

\subsection{Summary}

This research makes me more understand the flow how animation pipeline goes smoothly part by part before the animation is being bake into television and cinema or other devices. All animation methodology needs a solid structure to make it move to final concept then to good product. Before we go to industrial final training before going to the new world this research makes, we know, thus advantage and disadvantages, strength or weakness for making we easier to choose where we are going to go and from what to start and how to end. Too many knowledges that I have in this research. The real world is not a game, we are who is going to represent the future.

\section{REFERENCES}

1. (Williams, 2001)

2. http://www.thisdayindisneyhistory.com/NineOldMen.ht $\mathrm{ml}$

3. (Newsweek, October 21, 1999)

4. http://www.apa.org/science/about/psa/2011/05/facialexpressions.aspx

5. (LoBrutto, 1994)

6. https://en.wikipedia.org/wiki/Filmmaking

7. (Mediafreaks, 2003)

8. http://media-freaks.com/the-process-of-3d-animation/

9. (Jacob, 2010)

10. http://www.speakingofanimation.com/2010/05/bodylanguage-research/

11. (Williams, 2001)

12. Body language kin Anderson from nine old man

13. (Williams, 2001)

14. http://www.floobynooby.com/pdfs/The_Animators_Survi val_Kit.pdf

15. (Adbot, 2013)

16. https://en.wikipedia.org/wiki/Show,_don\%27t_tell

17. (Sajan, 1988)

18. http://www.viz.tamu.edu/courses/tutorials/sajan/

\section{BIBLIOGRAPHY}

Adbot, W. (2013, February 28). Wikepedia Show But Don't Tell Retrieved from https://en.wikipedia.org/wiki/Show,_don\%27t_tell

Jacob. (2010, May 05). Speaking Of Animation. Retrieved from http://www.speakingofanimation.com/2010/05/body-language-research/

LoBrutto, V. (1994). Sound-On-Film. Westport.

Mediafreaks. (2003). The Process of 3D Animation. Retrieved from http://media-freaks.com/the-process-of-3d-animation/

Newsweek. (October 21, 1999). In the blink of an eye.

Sajan, T. A. (1988). Retrieved from http://www.viz.tamu.edu/courses/tutorials/sajan/

Williams, R. (2001). The Animator Survival Kit's. United State: Faber And Faber. 